\title{
Mesoscopic modeling of corrosion processes: pitting morphology evolution
}

\author{
A. Taleb ${ }^{1}$, C. Vautrin-U1 ${ }^{2}$, H. Mendy ${ }^{2}$, J. Stafiej $^{3}$ \& A. Chausse ${ }^{2}$ \\ ${ }^{1}$ Laboratoire d'Electrochimie et de Chimie Analytique, \\ Université Pierre. et Marie Curie, CNRS UMR 7575, ENSCP, Paris, \\ France \\ ${ }^{2}$ Laboratoire Analyses et Modelisations pour la Biologie et \\ l'Environnement, Universite d'Evry Val d'Essonne, Evry, France \\ ${ }^{3}$ Institute of Physical Chemistry, Polish Academy of Sciences, Warsaw, \\ Poland
}

\begin{abstract}
We use cellular automata simulations at a mesoscopic scale to describe the phenomenon governing the morphology of pitting corrosion. We show that spontaneous separation of anodic and cathodic reaction zones plays a major role in determining the shape of the corroded space. The location of these zones versus the corroded surface is a stochastic event that influences strongly the direction of the corrosion propagation.

Keywords: cellular automata, corrosion, pitting morphology.
\end{abstract}

\section{Introduction}

The corrosion phenomena lead to a strong modification of the metal surface properties. Both physical and chemical aspects of the surface are affected by this change. Depending on the corrosive environment properties in terms of $\mathrm{pH}$, temperature, aggressive ion concentration and potential, the metal surface is covered by an oxide film or not. In certain conditions, the presence of the film leads to a passivation of the surface that is separation of the corrosive media from the metal greatly reducing the rate of corrosion. The local breakdown of this protecting layer induces local corrosion named pitting corrosion or crevice corrosion if the site is occluded. 
Pitting corrosion has been studied for several decades by many authors and all of them state that this kind of local corrosion takes place in several stages: pit nucleation or initiation, pit propagation and for certain conditions pit healing [1-4].

In the present study, we are interested in the pit morphology during the propagation stage. This morphology is documented experimentally and established to be connected to the material and the aggressive solution properties. Different authors have reported relations between the pitting corrosion and crystallographic properties of the corroded metal [5-7]. This relation was explained mainly by the metal atom coordination. In fact, metal at crystal defects have less bonds than in the rest of material and thus is more reactive [5-7]. Some other authors show that for strong salt solutions pits grow hemispherically but change to more saucer-like shape in a later stage which means that the ratio pit depth and pit width is time dependent [8-11]. They show also that this ratio is temperature dependent [8-11]. Theses results can be understood in term of diffusion processes. In fact, the pit depth increases according to $\mathrm{t}^{1 / 2}$ as expected for a diffusion controlled process [11]. The pit grows faster without cover when only the pit depth provides the diffusion barrier [11].

On the theoretical side local corrosion is a very complicated interfacial growth problem with transport, diffusion, bulk and heterogeneous chemical reactions coupled with each other and leading to specific surface phenomena such as passivation, depassivation and dissolution. The first principle atomic scale approaches to this phenomenon seem to be a difficult task. To understand and reproduce the rich phenomenology associated with corrosion system different approaches going from a mesoscopic to a macroscopic level were developed.

In our modelling we remain within the mesoscopic approaches which combine the macroscopic phenomenology with the stochastic character of the processes originating from the microscopic scale phenomena. Working at the mesoscopic scale we avoid the difficulties of the microscopic level and retain only the essential features of the studied phenomena.

The present work is a theoretical attempt to understand some characteristic of pitting morphology. We show using a simple model that we can reproduce and understand qualitatively some pitting morphologies. Hereafter we introduce anodic and cathodic reactions that can be separated in space. As a result of this separation heterogeneities in the distribution of $\mathrm{H}^{+}$and $\mathrm{OH}^{-}$ions can be formed [12]. Diffusion of these ions and their possible neutralization counteract the formation of these heterogeneities which determines the pit morphology. Since the pitting corrosion is a stochastic process in our model we use a stochastic cellular automaton (CA) as done in [12-17].

The paper is organized as follows. In section 2 we summarize the physicochemical model and its cellular automata representation. In section 3 we describe our simulations results with the corresponding discussion. In the final section we give some conclusions. 


\section{The model}

The model has been already presented elsewhere [12]. We recall here the points which are important for understanding of the new results presented in this work.

We consider a piece of metal material with a flat surface in contact with a corrosive environment. The surface is covered by an insulating layer (painted). The corrosion process starts from a single punctual damage of this layer. Then the role of the layer is to impose open circuit conditions with strict anodic and cathodic reaction balance inside the developing corrosion cavity. The metal corrosion is governed by a suite of surface local chemical and electrochemical reactions followed by bulk reactions. The metal anodic dissolution is followed by metal cation hydrolysis in acidic or neutral medium, with corrosion products detached from the surface.

$$
\mathrm{Me}+\mathrm{H}_{2} \mathrm{O} \rightarrow \mathrm{MeOH}_{\mathrm{aq}}+\mathrm{e}^{-}+\mathrm{H}^{+}
$$

Metal anodic oxidation in basic medium leads to the formation of corrosion products on the metal surface.

$$
\mathrm{Me}+\mathrm{OH}^{-} \rightarrow \mathrm{MeOH}_{\text {solid }}+\mathrm{e}^{-}
$$

Anodic reactions require simultaneous cathodic reactions. These are reduction of the hydrogen ion or water depending on the acidity of the environment.

$$
\begin{gathered}
\mathrm{H}^{+}+\mathrm{e}^{-} \rightarrow 1 / 2 \mathrm{H}_{2} \\
\mathrm{H}_{2} \mathrm{O}+\mathrm{e}^{-} \rightarrow 1 / 2 \mathrm{H}_{2}+\mathrm{OH}^{-}
\end{gathered}
$$

When the oxidation and reduction are spatially separated, thereafter named SSE reactions, anodic reactions (cathodic reactions) acidify (basify) the environment leading to $\mathrm{pH}$ inhomogeneity of the solution.

We study the effect of prevailing SSE reactions on the corrosion process. We take an implicit account of the presence of aggressive ions assuming that they may induce dissolution of $\mathrm{MeOH}_{\text {solid }}$

$$
\mathrm{MeOH}_{\text {solid }} \rightarrow \mathrm{MeOH}_{\mathrm{aq}}
$$

especially when the medium is acidic. We consider $\mathrm{MeOH}_{\mathrm{aq}}$ as a part of the environment solution.

The ions $\mathrm{H}^{+}$and $\mathrm{OH}^{-}$generated in the solution by SSE reactions diffuse and may neutralize one another:

$$
\mathrm{H}^{+}+\mathrm{OH}^{-} \rightarrow \mathrm{H}_{2} \mathrm{O}
$$

To represent the above physicochemical model in the framework of a cellular automat approach we have to use discrete lattice. The lattice sites are labelled in 
our model as M, R, P, E, A, C and W. E stands for the solution sites having the original $\mathrm{pH}$ level. A (C) stands for the solution sites with increased acidity (basicity). M, R and $\mathrm{P}$ stand for bulk metal, surface reactive and passive metal sites. Bulk metal sites cannot have E, A and $\mathrm{C}$ sites as nearest neighbours. Surface metal sites are those that have at least one solution site as a nearest neighbour. Reactive sites, R, can undergo anodic dissolution while the passive sites, P, cannot as explained further. Finally $\mathrm{W}$ are the isolating layer sites unchanged during the system evolution.

The transformation rules and probabilities associated with a given transformation path in our model depend on the local chemistry of the site. To characterize this local chemistry we use a simplified acidity scale - the difference of the numbers of acidic and basic sites, $\mathrm{N}^{\mathrm{exc}}=\mathrm{N}_{\mathrm{A}}-\mathrm{N}_{\mathrm{C}}$, in the nearest neighbourhood. Consequently we speak about acidic, neutral and basic environment for $\mathrm{N}^{\mathrm{exc}}>0, \mathrm{~N}^{\mathrm{exc}}=0$ and $\mathrm{N}^{\mathrm{exc}}<0$, respectively.

The anodic reactions (1) or (2) are represented by:

$$
\begin{gathered}
\mathrm{R} \rightarrow \mathrm{A} \\
\text { or } \mathrm{R}+\mathrm{C}(\mathrm{nn}) \rightarrow \mathrm{P}+\mathrm{E}(\mathrm{nn})
\end{gathered}
$$

where reaction (7) occurs when $\mathrm{N}^{\mathrm{exc}} \geq 0$ and reaction (8) otherwise. The "(nn)" indicates the nearest neighbour of the site transformed. The anodic reaction is possible if the site $R$ is connected with another surface site $S(S=R, P)$ by a path of nearest neighbours of types $\mathrm{M}, \mathrm{R}$ or $\mathrm{P}$ so that the cathodic counterpart of the reaction is possible according to one of the schemes corresponding to reactions (3) and (4) respectively

$$
\begin{aligned}
\mathrm{S}+\mathrm{A}(\mathrm{nn}) & \rightarrow \mathrm{S}+\mathrm{E}(\mathrm{nn}) \\
\mathrm{S}+\mathrm{E}(\mathrm{nn}) & \rightarrow \mathrm{S}+\mathrm{C}(\mathrm{nn})
\end{aligned}
$$

where reaction (9) occurs when $\mathrm{N}^{\mathrm{exc}} \geq 0$ and reaction (10) otherwise. If all neighbour sites are of type $\mathrm{C}$ the cathodic reaction cannot occur because we do not admit multiple $\mathrm{C}$ species on the same site and limit placing of the $\mathrm{C}$ site created in the nearest neighbour shell.

The reactions (7) - (9) correspond to SSE reactions. In our model we assume that these reactions occur with a probability $\mathrm{p}_{\mathrm{sse}}=1$. It can be lower due to the blocking of cathodic reaction discussed above.

We can mimic the dissolution of $\mathrm{MeOH}_{\text {solid }}$ (reaction 5) as

$$
\mathrm{P} \rightarrow \mathrm{E}
$$

The probability of this reaction is taken as $p_{P E}=0.25 \mathrm{~N}^{\mathrm{exc}}$ if $\mathrm{N}^{\mathrm{exc}} \geq 0$ and $\mathrm{p}_{\mathrm{PE}}=0$ otherwise. When an $\mathrm{R}$ or $\mathrm{P}$ site disappears, the $\mathrm{M}$ sites in contact with the solution become $\mathrm{R}$ sites. 
To account for diffusion $\mathrm{C}$ and $\mathrm{A}$ sites perform a random walk. The target site is selected at random from the nearest neighbours of the walker. If the target site is $\mathrm{E}$ the walker goes to it. If the walker is $\mathrm{C}$ and the target site is occupied by the walker A or vice versa both disappear to mimic reaction (6),

$$
\mathrm{A}+\mathrm{C} \rightarrow \mathrm{E}+\mathrm{E}
$$

Otherwise the random walker stays in its initial position. We set the diffusion rate with respect to the corrosion rate with $\mathrm{N}_{\mathrm{dif}}$ being an integer parameter indicating that we perform $\mathrm{N}_{\text {dif }}$ steps of random walk per one corrosion step. $\mathrm{N}_{\text {dif }}$ plays an important role in the corrosion evolution as diffusion counteracts the $\mathrm{pH}$ inhomogeneities created by SSE reactions.

We select to work with a square lattice in a $2 \mathrm{D}$ system for the computational efficiency reasons. The square lattice has 4 connectivity for random walk and metal site update rules. For the front connectivity we modify the rules. Two front sites are connected if they are von Neumann nearest neighbours or they have an $\mathrm{M}$ site as a common von Neumann neighbour. This prevents that a connected $\mathrm{M}$ area has a border composed of disconnected pieces.

The rules of evolution of the system are applied starting from an initial state on. The system is a square of $\mathrm{M}$ sites surrounded by inert $\mathrm{W}$ sites imitating isolating protective layer. Two $\mathrm{W}$ sites in the middle of the topmost line are turned into two $\mathrm{E}$ sites and the $\mathrm{M}$ two sites below become $\mathrm{R}$ sites to imitate the damage of the covering layer.

The simulation box sizes are from $1000 \times 1000$ to $1500 \times 1500$. Simulation runs up to several thousands steps are collected.

\section{Results and discussion}

The SSE reactions described above induce local $\mathrm{pH}$ inhomogeneities which in turn modify the corrosion process. The diffusion and neutralization of $\mathrm{H}^{+}$and $\mathrm{OH}^{-}$ions re-establishes the solution homogeneity.

\subsection{Snapshots}

In figure 1(a) and 1(b) we present the snapshots obtained for various simulation time steps. We distinguish two different behaviours:

First, at the early stage of simulation (figure 1(a)) the solution is locally neutral and the corrosion front uniformly rough. The corrosion front contains the same number of $\mathrm{R}$ and $\mathrm{P}$ sites.

Later (figure 1(b)) the separation of the solution (corrosion front) into acidic (smooth surface) and basic zones (rough surface) occurs with an intermediate neutral zone. Simultaneously the corroded space changes from hemispherical shape to complex asymmetrical one.

The transition from a homogeneous state to an inhomogeneous one for both the solution and the corrosion front is seen for $\mathrm{N}_{\text {dif }}$ ranging from 100 to 10000 . For small $\mathrm{N}_{\mathrm{dif}}$ this transition appears earlier. This suggests that for each $\mathrm{N}_{\mathrm{dif}}$ 
value there is a critical size of the corrosion cavity. Below this critical size the diffusion of $\mathrm{A}$ and $\mathrm{C}$ species is fast enough to neutralize of all of them. Above this critical size the diffusion is too slow for a complete neutralization.

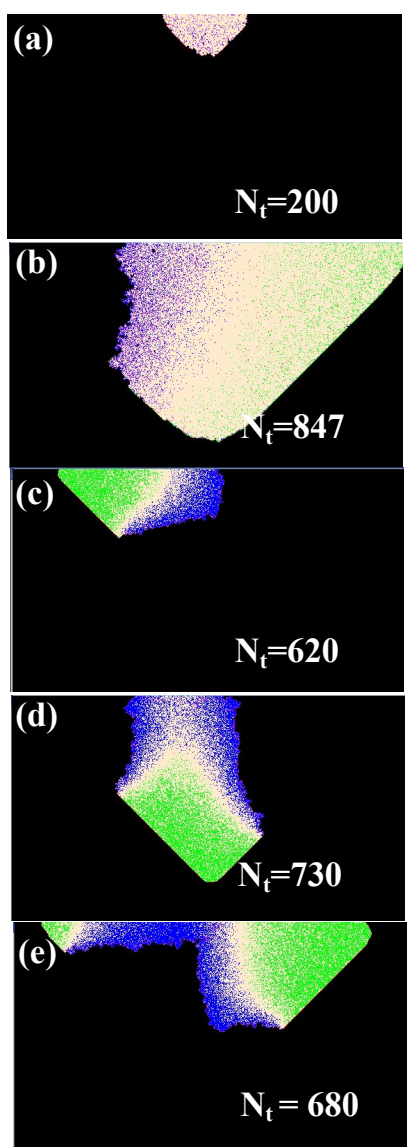

Figure 1: $\quad$ Snapshots corresponding to indicated simulation time steps $\left(\mathrm{N}_{\mathrm{t}}\right)$. (a) and (b) corresponds to the number of diffusion step $\mathrm{N}_{\text {dif }}=6000$ whereas (c), (d) and (e) to $\mathrm{N}_{\mathrm{dif}}=100$. The green, blue and white colours correspond respectively to Acidic, basic and neural zones.

The reproducibility of the simulation results has been tested on 10 independent runs with the same set of simulation parameters for each $\mathrm{N}_{\text {dif }}$.

The snapshots of figure 1 represent typical configurations of anodic and cathodic zones. In figure 1(b) and 1(c), the single anodic zone develops the corrosion either to the right side or to the left side respectively depending on a randomly created initial asymmetry. In figure 1(d) an in depth cavity develops downwards in contrast to the sideways cavities of figures 1(c) and 1(e). In figure 1(e) two anodic zones are separated by one cathodic zone. 
The difference observed in the cavity shapes of figures 1(b)-(e) outlines the stochastic character of the phenomena occurring during the corrosion process.

\subsection{Equivalent radius evolution}

In figure 2 we present the equivalent radius as a function of simulation time steps for several values of $\mathrm{N}_{\text {dif }}$. The equivalent radius is defined assuming that the total number of $\mathrm{M}$ species corroded, $\mathrm{N}_{\text {corr }}$ can be placed in a semicircle:

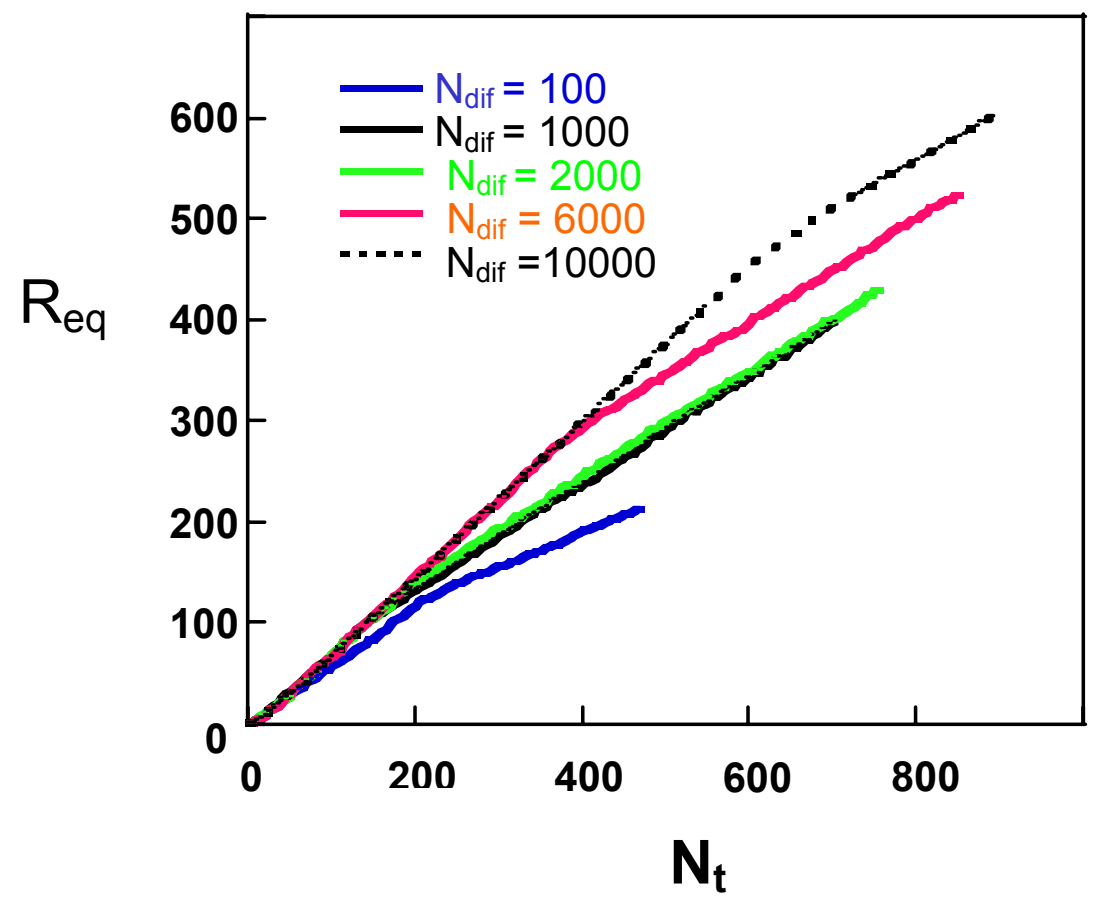

Figure 2: Evolution of the equivalent radius versus the simulation time steps, $\mathrm{N}_{\mathrm{t}}$, for indicated number of diffusion step $\mathrm{N}_{\text {dif. }}$.

$R_{e q}=\sqrt{2 N_{\text {corr }} / \pi}$ in the a length units. We use this equivalent radius because it depends linearly on time in the case of homogeneous development of the cavity as can be observed in early stage of simulation (figure 2).

In the initial stage of the corrosion the curves coincide and form a straight line for all $\mathrm{N}_{\text {dif }}$ value. After that the smaller $\mathrm{N}_{\text {dif }}$, the earlier the curve deviates from this line decreasing the slope. It coincides to zone separation. We call the first regime stationary regime (initial stage of the corrosion) and the second diffusion limited regime (later stage of the corrosion). 


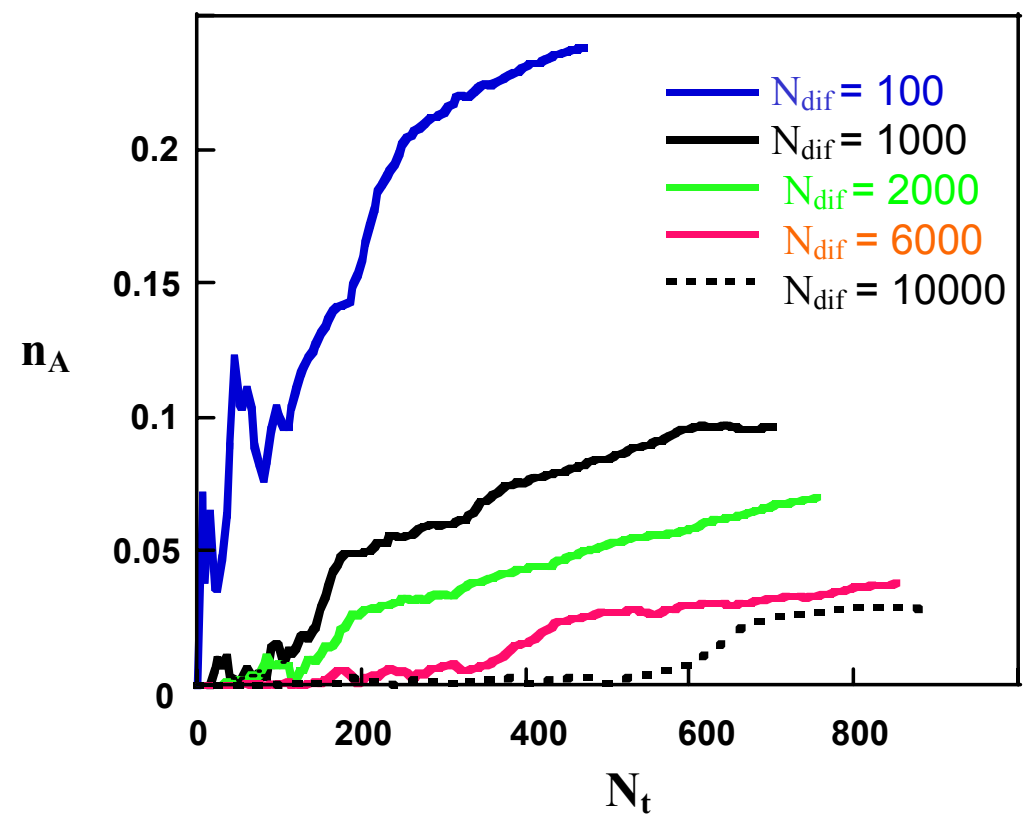

Figure 3: The evolution of acidic sites fraction, $\mathrm{n}_{\mathrm{A}}$, versus simulation time steps, $\mathrm{N}_{\mathrm{t}}$, for indicated number of diffusion step $\mathrm{N}_{\text {dif }}$.

In figure 3 we present the fraction of the number of A sites with respect to all the solution sites as a function of the time steps. A and $\mathrm{C}$ sites are created and disappear in pairs. Therefore the numbers of $\mathrm{A}$ and $\mathrm{C}$ sites are equal. The fraction of A sites is practically negligible until a certain time step where it begins to grow. It occurs later for larger $\mathrm{N}_{\text {dif. }}$. The increase of the fraction of $\mathrm{A}$ sites corresponds to the deviation of the $\mathrm{R}_{\mathrm{eq}} v s$. time step dependence from the stationary regime (figure 2). For the cavity larger than the critical size the diffusion is too slow for a complete neutralization of $\mathrm{A}$ and $\mathrm{C}$ sites. A and $\mathrm{C}$ sites survive in acidic and basic zones of the globally neutral solution. The fraction of A sites is smaller in the case of large $\mathrm{N}_{\text {dif }}$ because the critical cavity size is larger and contains already more E sites.

The transition from what we call stationary regime to diffusion limited regime is known in another model and observed in experimental characteristics of corrosion systems [18]. Here in the diffusion limited regime the cavity shapes are governed by an initial disposition of the zones resulting from a stochastic fluctuation.

\section{Conclusion}

We show that a simple mesoscopic stochastic model reproduces the main features observed in real experiments. The simulation results establish a clear connection between the geometrically complicated form of cavities and the 
solution inhomogeneity. Two types of separated zones appear with different $\mathrm{pH}$. The location of theses zones is a stochastic phenomenon influencing the morphology of the corroded area. We can see connection between the inhomogeneity of the corrosion process and the surface roughness.

\section{Acknowledgements}

The collaboration of our laboratories has been financially supported within PANCNRS project no. 14470 .

\section{References}

[1] Z. Szklarska-Smialowska, Pitting and Crevice Corrosion, (NACE International, 2005).

[2] D. Landoldt, Traité des Matériaux. Corrosion et Chimie de Surfaces de Matériaux. Presses Polytechniques et Universitaires Romandes, 1993, Lausanne.

[3] G. S. Frankel, J. Electrochem. Soc. 145, 2186 (1998).

[4] N. J. Laycock, S. P. White, J. Electrochem. Soc. 148 (7), B264 (2001).

[5] H. Kaesche, Werkstoffe Korr. 39, 152 (1988).

[6] M. Baumgartner and Kaesche, Corr. Sci. 29, 363 (1989).

[7] Lillard, R. S. Electrochem. Solid-state letters, 6(8), B29, (2003).

[8] J. Mankowski, Z. Szklarska-Smialowska, Corros. Sci. 15, 493, (1975).

[9] R. C. Alkire, K. P. Wong, Corros. Sci. 28, 411, (1988)

[10] W. Schwenk, Corrosion, 20, 129, (1964).

[11] P. Ernst, R. C. Newman Corro. Scie. 927, (2002).

[12] C. Vautrin-Ul, A. Chaussé, J. Stafiej and J. P. Badiali, Polish Journal of chemistry 78, 1795 (2004)

[13] P. Cordoba-Torres, R. P.Nogueira, L. De Miranda, L. Brenig, J. Wallenborn, V. Fairén, Electrochemica Acta, 46, 2975.

[14] A. Taleb, A. Chauss \'e, M. Dymitrowska, J. Stafiej, and J. P. Badiali, J. Phys. Chem. B 108, 952 (2004).

[15] J. Saunier, A. Chausse, J. Stafiej, and J. P. Badiali, J. Electroanal. Chem. 563, 239 (2004).

[16] J. Saunier, M. Dymitrowska, A. Chauss`'e, J. Stafiej, and J. P. Badiali, J. Electroanal. Chem. 582, 267 (2005).

[17] M. Malki, B. Baroux, Corro. Scie. 47, 171 (2005).

[18] L. Balazs, Phys. Rev B, 54, 1183, (1996). 